\title{
Triple-phase helical computed tomography in dogs with solid splenic masses
}

\author{
Kenji KUTARA ${ }^{1)}$, Mamiko SEKI ${ }^{1)}$, Kumiko ISHIGAKI ${ }^{1)}$, Kenji TESHIMA ${ }^{1)}$, \\ Chieko ISHIKAWA ${ }^{1)}$, Yumiko KAGAWA ${ }^{2)}$, Kazuya EDAMURA ${ }^{1 \text { ), }}$ \\ Tomohiro NAKAYAMA ${ }^{1)}$ and Kazushi ASANO ${ }^{1) *}$
1)Department of Veterinary Medicine, College of Bioresource Sciences, Nihon University, 1866 Kameino, Fujisawa, Kanagawa 252-0880, Japan
2) North Lab, 35-8-2 Hondoori Shiraishi, Sapporo, Hokkaido 003-0027, Japan

J. Vet. Med. Sci.

79(11): 1870-1877, 2017

doi: 10.1292/jvms.17-0253

Received: 8 May 2017

Accepted: 19 September 2017

Published online in J-STAGE:

7 October 2017

\begin{abstract}
We investigated the utility of triple-phase helical computed tomography (CT) in differentiating between benign and malignant splenic masses in dogs. Forty-two dogs with primary splenic masses underwent triple-phase helical CT scanning (before administration of contrast, and in the arterial phase, portal venous phase, and delayed phase) prior to splenectomy. Tissue specimens were sent for pathological diagnosis; these included hematomas ( $n=14)$, nodular hyperplasias $(n=12)$, hemangiosarcomas $(n=11)$, and undifferentiated sarcomas $(n=5)$. The CT findings were compared with the histological findings. Nodular hyperplasia significantly displayed a homogeneous normal enhancement pattern in all phases. Hemangiosarcoma displayed 2 significant contrast-enhancement patterns, including a homogeneous pattern of poor enhancement in all phases, and a heterogeneous remarkable enhancement pattern in the arterial and portal venous phases. Hematoma and undifferentiated sarcoma displayed a heterogeneous normal enhancement pattern in all phases. The contrast-enhanced volumetric ratios of hematoma tended to be greater than those of undifferentiated sarcoma. Our study demonstrated that the characteristic findings on triple-phase helical CT could be useful for the preoperative differentiation of hematoma, nodular hyperplasia, hemangiosarcoma, and undifferentiated sarcoma in dogs. Triple-phase helical CT may be a useful diagnostic tool in dogs with splenic masses.

KEY WORDS: computed tomography, dog, splenic hemangiosarcoma, splenic hematoma, splenic nodular hyperplasia
\end{abstract}

Solid splenic masses in dogs are important because of their high prevalence, and the high frequency of malignant tumors [9]. Approximately $51 \%$ of detected splenic masses are non-malignant, whereas the remaining $49 \%$ are malignant [14]. Nodular hyperplasia and hematoma account for approximately $80 \%$ of benign masses [13]. Hemangiosarcoma is the most common malignant lesion, accounting for $80 \%$ of splenic malignancies [19]. Accurate diagnosis is critical as malignant and non-malignant conditions have radically different prognoses. However, the morphological features and associated clinical signs of many splenic masses overlap [9]. Ultrasonography (US) and radiography are standard diagnostic tools used in the evaluation of splenic masses. Computed tomography may be used to investigate features of metastasis in the rest of the body. US findings in splenic masses are nonspecific, with overlapping features in benign and malignant masses [2, 3, 18, 19]. In addition, contrast-enhanced US has not proven useful in distinguishing splenic hemangiosarcoma from hematoma [4]. Therefore, the definitive diagnosis of a splenic mass is dependent on the pathological evaluation of the excised mass.

The advent of multi-detector helical computed tomography (MDCT) ensured that dynamic computed tomography (CT) could be used to evaluate the hemodynamics of hepatic masses, and that triple-phase helical CT could be used to scan the whole abdomen rapidly in 3 phases. In triple-phase helical CT, the images are obtained during the phase of preferential arterial enhancement, also known as the arterial phase, followed by the portal venous phase, and delayed phase images after a single bolus injection [8, 11]. Previous studies in humans and dogs have shown that triple-phase helical CT improves the detection of various hepatic masses [1, $5,11,12]$. In addition, 3-dimensional (3D) reconstructions of triple-phase helical CT can be useful for preoperative planning. To the best of our knowledge, there has been no report on the use of MDCT to evaluate the hemodynamics of splenic masses using dynamic CT and triple-phase helical CT.

The aim of this study was to characterize triple-phase CT findings in naturally-occurring hematoma, nodular hyperplasia,

*Correspondence to: Asano, K.: asano.kazushi@nihon-u.ac.jp

O2017 The Japanese Society of Veterinary Science

This is an open-access article distributed under the terms of the Creative Commons Attribution Non-Commercial No Derivatives (by-nc-nd) License. (CC-BY-NC-ND 4.0: https://creativecommons.org/licenses/by-nc-nd/4.o/) 
hemangiosarcoma, and undifferentiated sarcoma. We believe that triple-phase helical CT is useful for the diagnosis and characterization of splenic masses.

\section{MATERIALS AND METHODS}

\section{Animals}

The medical records of the Animal Medical Center of Nihon University were investigated retrospectively from 2007 to 2014. The criteria for the selection of cases were as follows: (1) Dogs that underwent preoperative triple-phase helical CT; (2) Dogs with surgical removal of the spleen and histopathological examination of the specimen by a pathologist (Y K); (3) the numbers of the same histopathological masses were greater than 2. Forty-two dogs could be included according to the above criteria. Of the 42 dogs (24 male and 18 female dogs; aged 3-16 years) with splenic masses, 14, 12, 11 and 5 dogs were diagnosed with hematoma, nodular hyperplasia, hemangiosarcoma, and undifferentiated sarcoma, respectively. On histopathological examination, all cases of hematoma were derived from nodular hyperplasia or extra-medullary hematopoiesis. Two cases of undifferentiated sarcoma included nodular hyperplasia and malignant splenic stromal neoplasia. The breeds were as follows: 7 golden retrievers, 7 mixed-breed dogs, 4 Labrador retrievers, 3 Pembroke Welsh corgis, 3 beagles, 3 miniature dachshunds, 2 English cocker spaniels, 2 Maltese poodles, 1 American cocker spaniel, 1 Cavalier King Charles spaniel, 1 Chihuahua, 1 flat-coated retriever, 1 French bulldog, 1 miniature schnauzer, 1 miniature poodle, 1 pug, 1 Polish lowland sheepdog, 1 Shetland sheepdog and 1 toy poodle. The median body weight was $12.5 \mathrm{~kg}$ (range: $2.4-37.2 \mathrm{~kg}$ ).

\section{Triple-phase helical CT technique}

In all dogs, an 18-27 G over-the-needle catheter was inserted in the cephalic vein. Each dog was pre-medicated with midazolam hydrochloride $(0.2 \mathrm{mg} / \mathrm{kg}$, intravenously) and butorphanol tartrate $(0.2 \mathrm{mg} / \mathrm{kg}$, intravenously), and intubated following induction with intravenous propofol. General anesthesia was maintained by mechanical ventilation with isoflurane (1.5-2\%) and oxygen (2 $l / \mathrm{min}$ ). All dogs were positioned in ventral recumbency, and all scans were obtained with a 16-slice MDCT scanner (Aquilion 16; Toshiba Medical Systems, Otawara, Japan). The scanning parameters were as follows: rotation time $=0.5$ sec; slice thickness $=1-2$ $\mathrm{mm}$; reconstruction interval $=0.5-1 \mathrm{~mm}$; table speed $=16-32 \mathrm{~mm} /$ rotation; helical pitch $=16.0 ; \mathrm{X}$-ray tube voltage $=120 \mathrm{kV}$; and $\mathrm{X}$-ray tube current $=150 \mathrm{~mA}$. All helical scans were started at the tip of the wing of the ilium, proceeded in a cranial direction, and covered the entire liver. Iohexol (Ioverin 300; Teva Pharma Japan Inc., Nagoya, Japan) was used as a contrast medium and was administered at a dose of $2.5 \mathrm{~m} / \mathrm{kg}$ (750 mg iodine $/ \mathrm{kg}$ ) via the cephalic vein with a power injector (Auto Enhance A-60; NemotoKyorindo, Tokyo, Japan). The injection time was fixed at $15-20 \mathrm{sec}$ (injection speed $=0.3-3 \mathrm{ml} / \mathrm{sec}$ ) as described previously [6-8, 16]. In cases in which the injection speed was calculated to exceed $3 \mathrm{ml} / \mathrm{sec}$ with the injection time of the contrast medium within $20 \mathrm{sec}$, the injection speed was fixed at $3 \mathrm{~m} / / \mathrm{sec}$ (and the range of injection time was 21-31 sec). Pre-contrast (before the injection of contrast medium), arterial phase ( $20 \mathrm{sec}$ after the start of injection of the contrast medium), portal venous phase (40 sec after the start of the injection), and delayed phase (120 sec after the start of the injection) scans were obtained as described previously [6-8, $16]$.

The scanning time for each phase was approximately $8-12 \mathrm{sec}$ for all subjects. In all cases, 3D images were reconstructed on a workstation after scanning (AZE Virtual Place Plus; AZE, Tokyo, Japan).

\section{Image analysis}

All CT images were reviewed on the workstation. The size of the lesion was measured from the image. The contrast values were represented in Hounsfield units (HU). Contrast values within the mass were measured in the area of brightest contrast (maximum contrast value) and the area of least contrast (minimum contrast value). For comparative purposes, the contrast values in 3 portions of the remaining normal splenic parenchyma adjacent to the mass were measured, and averaged to represent the contrast value of the splenic parenchyma. A region-of-interest (ROI) of approximately $30 \mathrm{~mm}^{2}$ was used, whenever possible, for all measurements of contrast values. The contrast values of the splenic parenchyma and the mass were used to calculate the ratio of mass enhancement using the following formula:

Mass enhancement $(\%)=$ Maximum contrast value (mass)/Mean contrast value (spleen) $\times 100(1)$

The contrast-enhancement pattern was defined by the combination of the degree of enhancement and the enhancement homogeneity. 'Remarkable enhancement' was defined as a percentage of mass enhancement greater than $200 \%$. 'Normal enhancement' was defined as a percentage of mass enhancement less than $200 \%$, and a maximum contrast value measuring over $60 \mathrm{HU}$, which was equivalent to the mean pre-contrast CT value of splenic parenchyma in this study and as described in previous study [17]. 'Poor enhancement' was defined as a maximum contrast value measuring less than $60 \mathrm{HU}$. A lesion was defined as 'homogeneous' if the maximum and minimum intensities were observed within the same mass enhancement categories. If they were not observed within the same mass enhancement categories, the cases were defined as 'heterogeneous'. In the cases with heterogeneous enhancement, 3D images were reconstructed in each phase to determine the volume of the enhancement area in the mass (Fig. 1). The volume of the area within the mass with a contrast value of greater than $60 \mathrm{HU}$, and the total mass volume were used to calculate the enhanced volumetric ratio, using the following formula:

Enhanced volumetric ratio $=$ Volume $\geq 60 \mathrm{HU} /$ Mass volume $\times 100(2)$ 

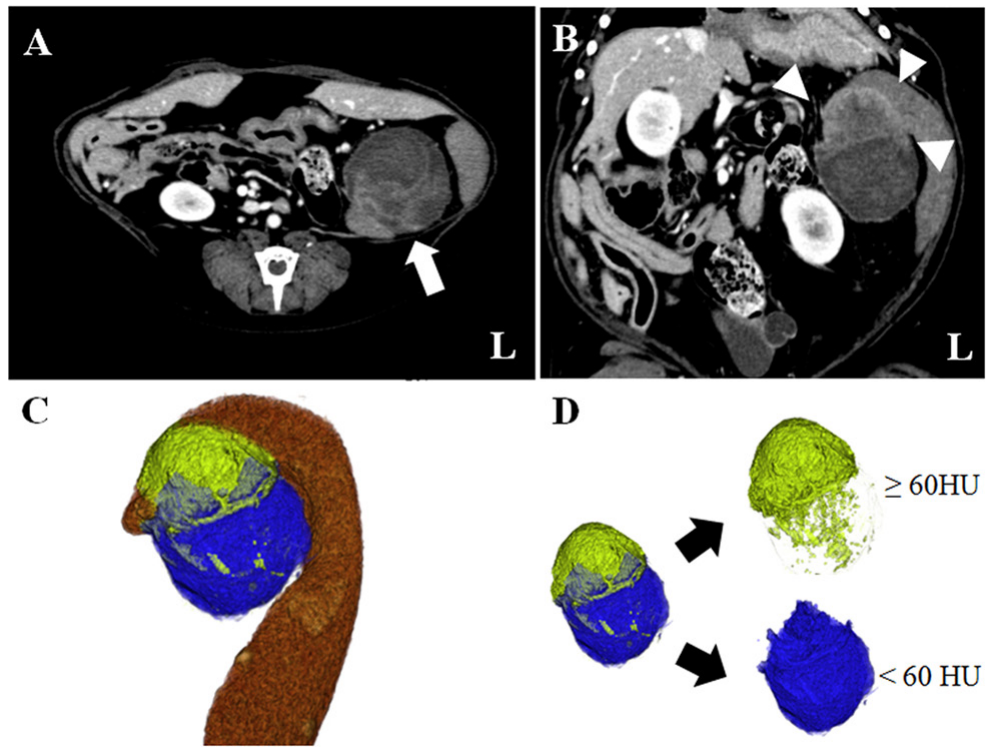

Fig. 1. Three-dimensional image reconstructed for calculation of enhanced volumetric ratio. (A) Transverse contrast-enhanced CT image shows a mass in the spleen (arrow). The contrast-enhancement pattern was heterogeneous iso-enhancement. (B) Coronal contrast-enhanced CT image shows the splenic mass. A portion (arrow head) of more than 60 Hounsfield units (HU) was located in the cranial aspect of the mass. (C) Threedimensional image of the spleen including mass. The yellow portion is the $\geq 60 \mathrm{HU}$ portion of the mass, and the blue portion the $<60 \mathrm{HU}$ portion; the brown portion is splenic parenchyma. (D) The images separated into $\geq 60$ and $<60 \mathrm{HU}$ areas.

\section{Data analysis}

Statistical tests were performed using commercially available statistical analysis software (Stat Mate III; ATMS Co., Tokyo, Japan). Data regarding the splenic mass and parenchymal contrast values, and the pre-contrast CT values were represented as mean \pm standard deviation. The enhanced volumetric ratio, body weight, and mass size were represented as the median (range). Spearman's rank test was used to analyze the relationships between all the contrast values, and body weight, mass size, enhanced volumetric ratio, and injection speed of contrast medium. The Mann-Whitney $U$ test was used to compare the enhanced splenic parenchyma and mass contrast values. The contrast-enhancement patterns were analyzed using the chi-square test, followed by the Yates post hoc correction test. The Kruskal-Wallis H test was used to compare the enhanced volumetric ratio, ratio of mass enhancement, size, pre-contrast CT values, and contrast values among each mass type. A $P$ value of less than 0.05 was considered to indicate a statistically significant difference.

\section{RESULTS}

The median mass size was 62.5 (range: 8.6-217.7) $\mathrm{mm}$. The median mass size of hematoma, nodular hyperplasia, hemangiosarcoma, and undifferentiated sarcoma was 76.5 (range: 25.5-217.7), 19.5 (range: 8.6-145.5), 97 (range: 33.1-164.4), and 61.7 (range: 50.2-169.1) $\mathrm{mm}$, respectively. The mass size of nodular hyperplasia was significantly smaller than that of hematoma and hemangiosarcoma $(P=0.022)$.

The mean pre-contrast CT values in hematoma, nodular hyperplasia, hemangiosarcoma, undifferentiated sarcoma, and surrounding normal splenic parenchyma were 54.2 $\pm 11.7 \mathrm{HU}, 56.4 \pm 13.0 \mathrm{HU}, 35.3 \pm 11.9 \mathrm{HU}, 45.6 \pm 11.8 \mathrm{HU}$, and $58.8 \pm$ $9.9 \mathrm{HU}$, respectively. The pre-contrast CT value of hemangiosarcoma was significantly lower than those of hematoma $(P<0.05)$, nodular hyperplasia $(P<0.05)$, and surrounding normal splenic parenchyma $(P<0.01)$.

The contrast values of the splenic parenchyma and mass, and the ratios of mass enhancement in each phase, are summarized in Tables 1-3. The maximum contrast value of the mass and the ratio of mass enhancement did not differ significantly between the mass groups. The minimum contrast values of hematoma and hemangiosarcoma were significantly lower than those of nodular hyperplasia in the arterial $(P=0.012)$, portal venous $(P=0.017)$, and delayed $(P=0.011)$ phases. The contrast values of the mass and the surrounding splenic parenchyma had no significant correlation with body weight, mass size, or injection speed.

The contrast-enhancement patterns of the splenic masses are summarized in Table 4 . The heterogeneous normal enhancement pattern significantly predominated, compared to the other patterns in all phases of the hematoma cases $(12 / 14$ cases, $P<0.001$; Fig. 2). In addition, the homogeneous normal enhancement pattern was significantly predominant, when compared with the other patterns in all phases of the nodular hyperplasia cases $(9 / 12$ cases, $P<0.001$; Fig. 3$)$. Of 11 hemangiosarcoma cases, 6 showed a homogeneous poor enhancement pattern in all phases $(P<0.001$; Fig. 4$)$, and the other 3 displayed a heterogeneous remarkable enhancement pattern in the arterial and portal venous phases $(P=0.0025$; Fig. 5). All undifferentiated sarcoma cases showed a heterogeneous normal enhancement pattern in all phases $(P=0.014$; Fig. 6$)$. 
Table 1. The mean contrast values of hematoma, NH, hemangiosarcoma and undifferentiated sarcoma as seen in 42 patients on $\mathrm{CT}$ scans during AP

\begin{tabular}{|c|c|c|c|c|c|}
\hline \multirow{3}{*}{ Type } & \multirow{3}{*}{$\mathrm{n}$} & \multicolumn{4}{|c|}{ Contrast value in AP (HU) } \\
\hline & & \multicolumn{2}{|c|}{ Mass } & \multirow{2}{*}{ Parenchyma } & \multirow{2}{*}{$\begin{array}{l}\text { The ratio of mass } \\
\text { enhancement }(\%)\end{array}$} \\
\hline & & Maximum & Minimum & & \\
\hline Hematoma & 14 & $84.8 \pm 15.2$ & $33.5 \pm 17.5$ & $83.6 \pm 15.5$ & $105.2 \pm 21.7$ \\
\hline $\mathrm{NH}$ & 12 & $86.9 \pm 16.5$ & $65.1 \pm 30.3$ & $88.5 \pm 23.2$ & $103.8 \pm 28.3$ \\
\hline Hemangiosarcoma & 11 & $127.6 \pm 141.1$ & $32.8 \pm 7.5$ & $86.3 \pm 20.2$ & $159.9 \pm 177.8$ \\
\hline Undifferentiated sarcoma & 5 & $80.1 \pm 15.5$ & $30.0 \pm 14.8$ & $83.9 \pm 13.2$ & $96.2 \pm 17.1$ \\
\hline
\end{tabular}

The ratio of mass enhancement was calculated by the ratio of maximum contrast value of mass to the contrast value of splenic parenchyma. AP, arterial phase; HU, Hounsfield Units, NH, nodular hyperplasia.

Table 2. The mean contrast values of hematoma, NH, hemangiosarcoma, and undifferentiated sarcoma as seen in 42 patients on CT scans during PVP

\begin{tabular}{|c|c|c|c|c|c|}
\hline \multirow{3}{*}{ Type } & \multirow{3}{*}{$\mathrm{n}$} & \multicolumn{4}{|c|}{ Contrast value in PVP (HU) } \\
\hline & & \multicolumn{2}{|c|}{ Mass } & \multirow{2}{*}{ Parenchyma } & \multirow{2}{*}{$\begin{array}{l}\text { The ratio of mass } \\
\text { enhancement (\%) }\end{array}$} \\
\hline & & Maximum & Minimum & & \\
\hline Hematoma & 14 & $102.1 \pm 18.2$ & $38.4 \pm 23.5$ & $102.0 \pm 19.5$ & $106.4 \pm 30.0$ \\
\hline NH & 12 & $113.6 \pm 22.8$ & $78.3 \pm 38.1$ & $109.2 \pm 18.3$ & $106.7 \pm 26.8$ \\
\hline Hemangiosarcoma & 11 & $113.5 \pm 120.0$ & $34.7 \pm 9.9$ & $109.0 \pm 30.7$ & $114.0 \pm 121.7$ \\
\hline Undifferentiated sarcoma & 5 & $84.4 \pm 15.4$ & $36.2 \pm 14.2$ & $98.8 \pm 13.5$ & $88.3 \pm 27.2$ \\
\hline
\end{tabular}

The ratio of mass enhancement was calculated by the ratio of maximum contrast value of mass to the contrast value of splenic parenchyma. HU, Hounsfield Units, NH, nodular hyperplasia; PVP, portal venous phase.

Table 3. The mean contrast values of hematoma, NH, hemangiosarcoma, and undifferentiated sarcoma as seen in 42 patients on $\mathrm{CT}$ scans during DP

\begin{tabular}{|c|c|c|c|c|c|}
\hline \multirow{3}{*}{ Type } & \multirow{3}{*}{$\mathrm{n}$} & \multicolumn{4}{|c|}{ Contrast value in DP (HU) } \\
\hline & & \multicolumn{2}{|c|}{ Mass } & \multirow{2}{*}{ Parencyma } & \multirow{2}{*}{$\begin{array}{l}\text { The ratio of mass } \\
\text { enhancement }(\%)\end{array}$} \\
\hline & & Maximum & Minimum & & \\
\hline Hematoma & 14 & $109.1 \pm 12.4$ & $39.3 \pm 24.6$ & $104.8 \pm 11.1$ & $106.0 \pm 17.6$ \\
\hline $\mathrm{NH}$ & 12 & $112.4 \pm 18.5$ & $77.5 \pm 39.8$ & $104.9 \pm 16.1$ & $107.3 \pm 8.5$ \\
\hline Hemangiosarcoma & 11 & $82.7 \pm 60.0$ & $33.9 \pm 8.2$ & $105.7 \pm 24.4$ & $82.3 \pm 63.3$ \\
\hline Undifferentiated sarcoma & 5 & $79.4 \pm 11.5$ & $39.1 \pm 12.4$ & $104.9 \pm 6.3$ & $76.2 \pm 14.3$ \\
\hline
\end{tabular}

The ratio of mass enhancement was calculated by the ratio of maximum contrast value of mass to the contrast value of splenic parenchyma. DP, delayed phase; HU, Hounsfield Units, NH, nodular hyperplasia.

Table 4. Characteristic contrast enhancement pattern seen in 42 splenic masses

\begin{tabular}{|c|c|c|c|c|c|c|c|}
\hline \multirow{2}{*}{ Type } & \multirow{2}{*}{$\mathrm{n}$} & \multirow{2}{*}{ Contrast } & \multicolumn{4}{|c|}{ Enhancement } & \multirow{2}{*}{$(\%)$} \\
\hline & & & AP & PVP & DP & $\mathrm{n}$ & \\
\hline \multirow[t]{2}{*}{ Hematoma } & 14 & homogeneous & normal & normal & normal & 2 & 14.3 \\
\hline & & heterogeneous & normal & normal & normal & 12 & $85.7^{\text {a) }}$ \\
\hline \multirow[t]{2}{*}{$\mathrm{NH}$} & 12 & homogeneous & normal & normal & normal & 9 & $75.0^{\mathrm{a})}$ \\
\hline & & heterogeneous & normal & normal & normal & 3 & 25.0 \\
\hline \multirow[t]{3}{*}{ Hemangiosarcoma } & 11 & homogeneous & poor & poor & poor & 6 & $54.5^{\text {a) }}$ \\
\hline & & heterogeneous & remarkable & remarkable & normal & 3 & $27.3^{\mathrm{a})}$ \\
\hline & & heterogeneous & normal & normal & normal & 2 & 18.2 \\
\hline Undifferentiated sarcoma & 5 & heterogeneous & normal & normal & normal & 5 & $100.0^{\mathrm{a})}$ \\
\hline
\end{tabular}

AP, arterial phase; DP, delayed phase; $\mathrm{NH}$, nodular hyperplasia; PVP, portal venous phase; a), significantly larger compared with the other groups $(P<0.05)$.

The enhanced volumetric ratios of splenic masses are summarized in Table 5. In all phases, the enhanced volumetric ratio of hemangiosarcoma was significantly lower than that of hematoma and nodular hyperplasia $(P<0.001)$. The hemangiosarcoma cases with remarkable enhancement in the arterial and portal venous phases showed high enhanced volumetric ratios, whereas the 

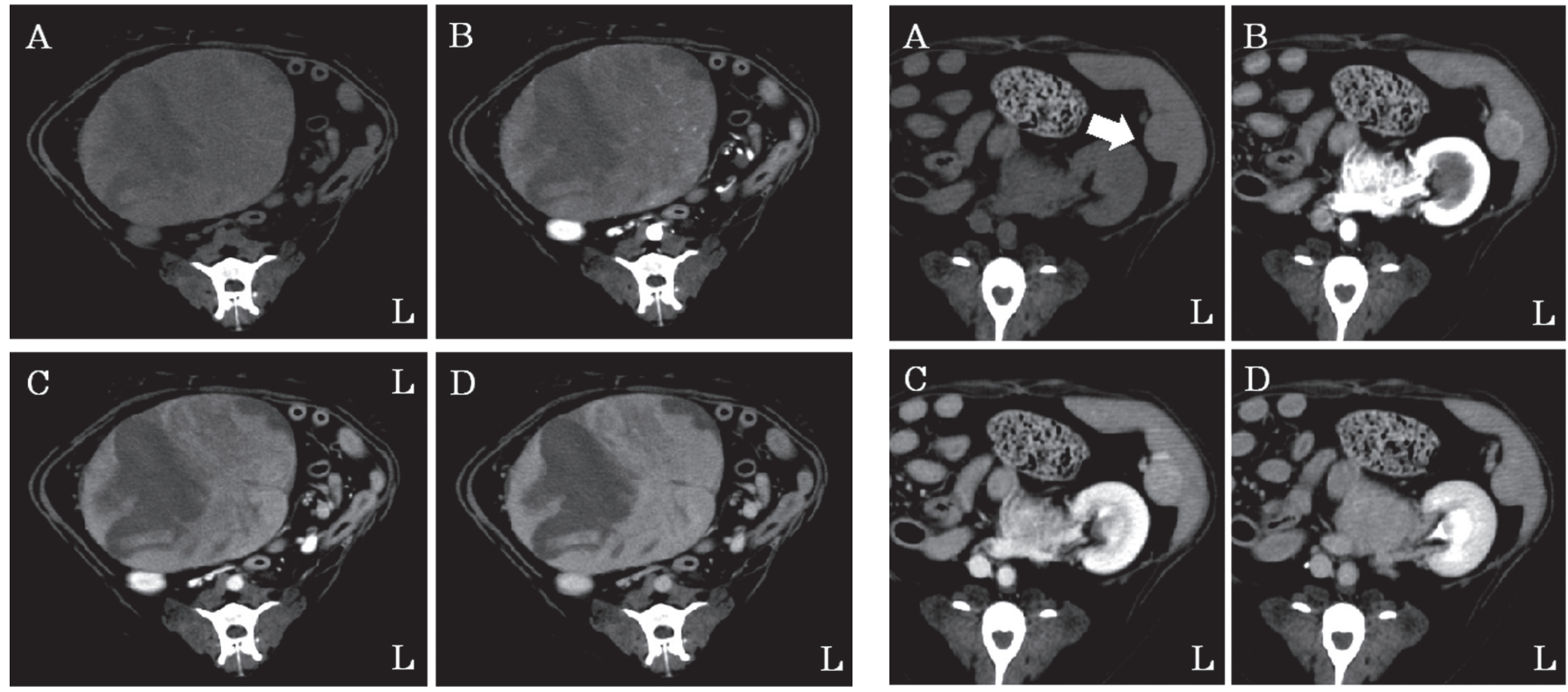

Fig. 2. Contrast-enhancement pattern in a patient with hematoma. (A) Transverse pre-contrast CT image showing a mass. (B) Heterogeneous normal enhancement in the arterial phase. The enhanced volumetric ratio was $61.9 \%$. (C) Heterogeneous normal enhancement in the portal venous phase. The enhanced volumetric ratio was $75.9 \%$. (D) Heterogeneous normal enhancement in the delayed phase. The enhanced volumetric ratio was $80.9 \%$.

Fig. 3. Contrast-enhancement pattern in a patient with nodular hyperplasia. (A) Transverse pre-contrast CT image showing a mass in the spleen (arrow). (B) Homogeneous normal enhancement in the arterial phase. The ratio of mass enhancement was $121.1 \%$. (C) Homogeneous normal enhancement in the portal venous phase. The ratio of mass enhancement was $136.2 \%$. (D) Homogeneous normal enhancement in the delayed phase. The ratio of mass enhancement was $105.1 \%$.
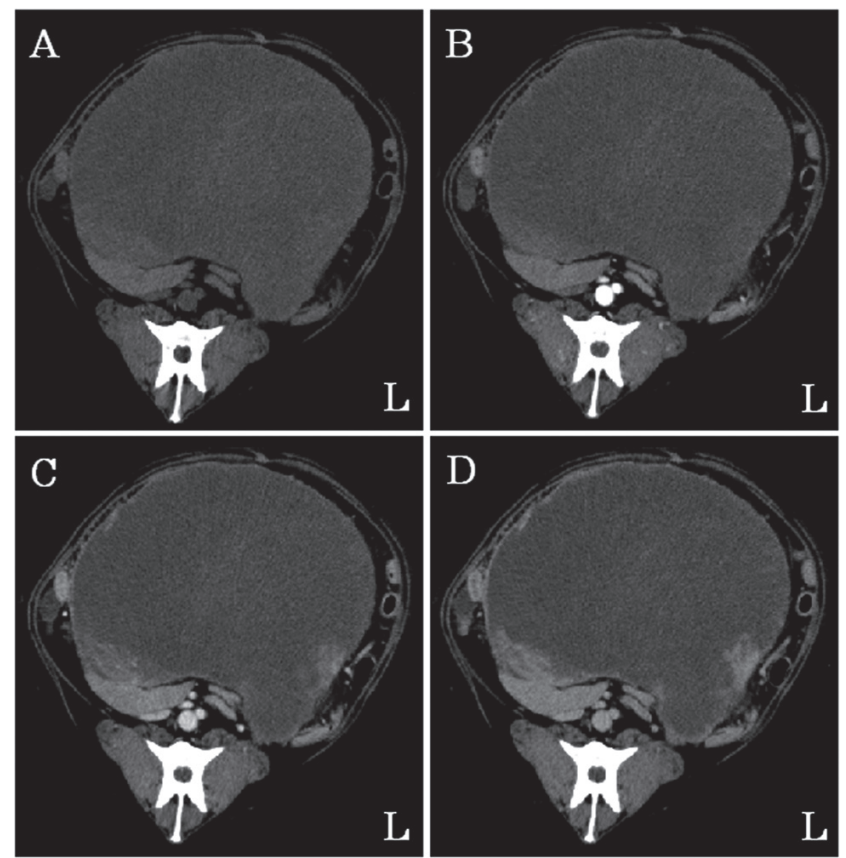

Fig. 4. Contrast-enhancement pattern in a patient with hemangiosarcoma. (A) Transverse pre-contrast CT image showing a mass. (B) Homogeneous poor enhancement in the arterial phase. (C) Homogeneous poor enhancement in the portal venous phase. (D) Homogeneous poor enhancement in the delayed phase.
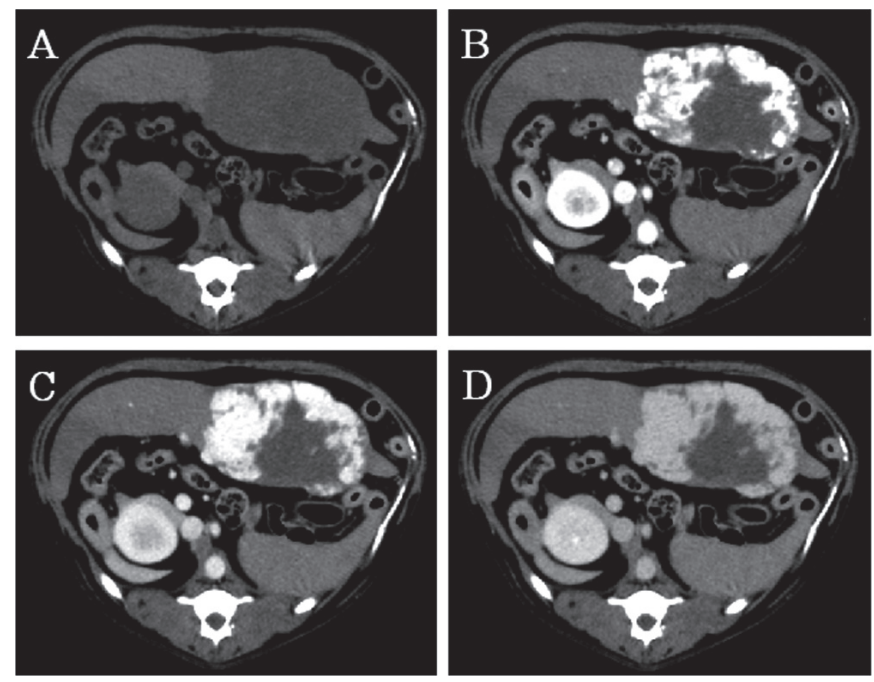

Fig. 5. Contrast-enhancement pattern in a patient with hemangiosarcoma. (A) Transverse pre-contrast CT image showing a mass. (B) Heterogeneous remarkable enhancement in the arterial phase. The enhanced volumetric ratio was $53.6 \%$, and the ratio of mass enhancement was $456.5 \%$. (C) Heterogeneous remarkable enhancement in the portal venous phase. The enhanced volumetric ratio was $62.1 \%$, and the ratio of mass enhancement was $310.9 \%$. (D) Heterogeneous normal enhancement in the delayed phase. The enhanced volumetric ratio was $73.6 \%$, and the ratio of mass enhancement was $143.8 \%$. 

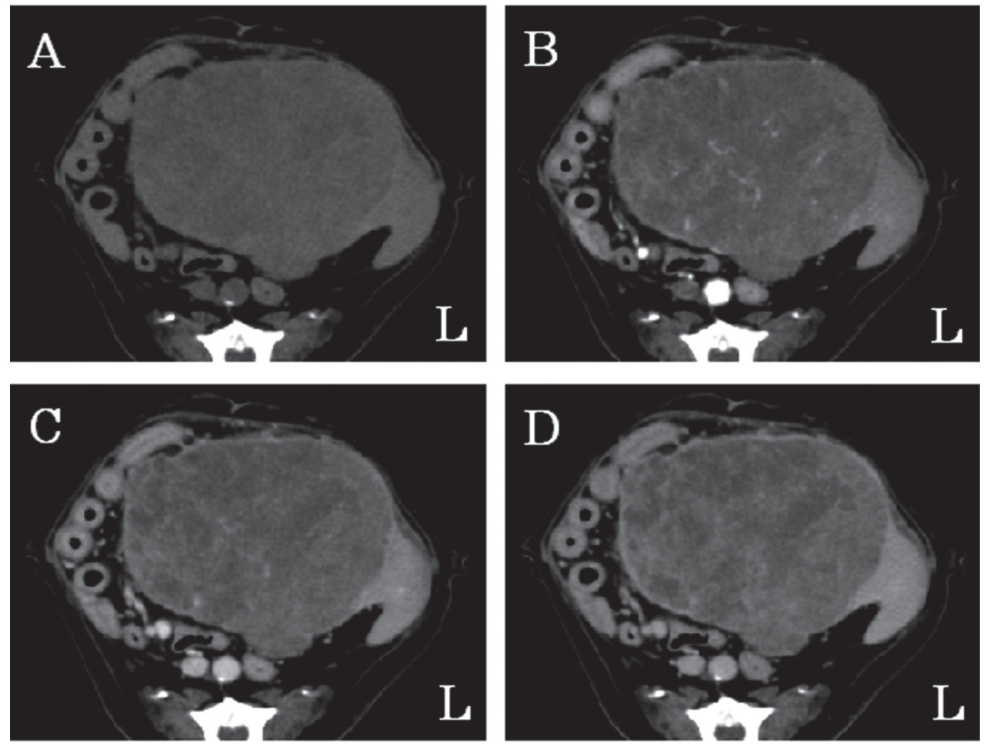

Fig. 6. Contrast-enhancement pattern in a patient with undifferentiated sarcoma. (A) Transverse pre-contrast CT image showing a mass. (B) Heterogeneous normal enhancement in the arterial phase. The enhanced volumetric ratio was $6.9 \%$. (C) Heterogeneous normal enhancement in the portal venous phase. The enhanced volumetric ratio was $11.8 \%$. (D) Heterogeneous normal enhancement in the delayed phase. The enhanced volumetric ratio was $16.1 \%$.

Table 5. The enhanced volumetric ratios of hematoma, NH, hemangiosarcoma, and undifferentiated sarcoma as seen in 42 patients on CT scans during AP, PVP and DP

\begin{tabular}{|c|c|c|c|c|c|c|c|}
\hline \multirow{2}{*}{ Type } & \multirow{2}{*}{$\mathrm{n}$} & \multicolumn{2}{|c|}{ AP (\%) } & \multicolumn{2}{|c|}{ PVP (\%) } & \multicolumn{2}{|c|}{ DP $(\%)$} \\
\hline & & Median & Range & Median & Range & Median & Range \\
\hline Hematoma & 14 & 47.5 & $(100.0-5.7)$ & 55.1 & $(100.0-9.1)$ & 57.7 & $(100.0-20.0)$ \\
\hline NH & 12 & 100.0 & $(100.0-47.8)$ & 100.0 & $(100.0-49.7)$ & 100.0 & $(199.9-50.6)$ \\
\hline Hemangiosarcoma & 11 & $0.0^{\mathrm{a}, \mathrm{b})}$ & $(68.9-0.0)$ & $0.0^{\mathrm{a}, \mathrm{b})}$ & $(80.9-0.0)$ & $0.0^{\mathrm{a}, \mathrm{b})}$ & $(92.3-0.0)$ \\
\hline Remarkable renhancement & 3 & 53.8 & $(68.9-18.9)$ & 62.1 & $(80.9-23.7)$ & 72.7 & $(92.3-55.5)$ \\
\hline Normal and poor enhancement & 8 & $0.0^{\mathrm{a}, \mathrm{b})}$ & $(10.8-0.0)$ & $0.0^{\mathrm{a}, \mathrm{b})}$ & $(16.5-0.0)$ & $0.0^{\mathrm{a}, \mathrm{b})}$ & $(25.7-0.0)$ \\
\hline Undifferentiated sarcoma & 5 & 21.3 & $(86.0-4.7)$ & 30.2 & $(94.0-11.9)$ & 48.8 & $(96.4-16.0)$ \\
\hline Pure undifferentiated sarcoma & 3 & $7.0^{\mathrm{b})}$ & $(21.3-4.7)$ & 22.3 & $(30.2-11.9)$ & 28.8 & $(48.8-16.0)$ \\
\hline Contained nodular hyperplasia & 2 & 83.1 & $(86.0-80.1)$ & 88.7 & $(94.0-83.3)$ & 91.3 & $(96.4-86.2)$ \\
\hline
\end{tabular}

AP, arterial phase; DP, delayed phase; NH, nodular hyperplasia; PVP, portal venous phase; a), significantly lower than hematoma $(P<0.05)$; b), significantly lower than nodular hyperplasia $(P<0.05)$.

hemangiosarcoma cases with normal and poor enhancement in all phases revealed low enhanced volumetric ratios. Two cases with co-existing undifferentiated sarcoma and nodular hyperplasia in the masses had high enhanced volumetric ratios. The enhanced volumetric ratios of the other 3 cases were significantly lower than those of nodular hyperplasia in the arterial phase $(P<0.001)$.

\section{DISCUSSION}

Triple-phase helical CT enabled the differentiation of splenic masses by analyzing the contrast-enhancement pattern. The characteristic CT finding of nodular hyperplasia was a homogeneous normal enhancement pattern in all phases. Hemangiosarcoma was characterized by 2 contrast-enhancement patterns, including a heterogeneous remarkable enhancement pattern in the arterial and portal venous phases, and a homogeneous poor enhancement pattern in all phases. A heterogeneous normal enhancement pattern was observed in cases with hematoma and undifferentiated sarcoma. The cases of hematoma had a tendency to show a high enhanced volumetric ratio value, whereas 3 of the 5 undifferentiated sarcoma cases without nodular hyperplasia had a tendency to show a low value. However, it was impossible to differentiate the other 2 undifferentiated sarcomas with nodular hyperplasia from hematoma.

On the histopathological examination, all cases of hematoma were derived from nodular hyperplasia or extra-medullary hematopoiesis. A lack of contrast enhancement of the mass has been reported in canine patients with splenic hematomas secondary to trauma, and in experimentally induced splenic hematomas in dogs $[2,10]$. In this study, hematoma displayed heterogeneous contrast enhancement. Thus, it is suggested that the regions of contrast enhancement in hematoma possibly represented the extant 
splenic parenchyma or nodular hyperplasia. In addition, the regions of poor contrast enhancement represented a hematocyst and necrotic tissue. In this study, the patients with hematoma had no history of abdominal trauma, as was the case in a previous study [2]. These findings may indicate that canine splenic hematoma results from bleeding in pre-existing nodular hyperplasia, or extramedullary hematopoiesis.

The hemangiosarcoma cases with a heterogeneous remarkable enhancement pattern showed the highest enhancement in the arterial phase. This phenomenon may imply that the blood supply for hemangiosarcoma is directly from the splenic artery. The homogeneous poor enhancement pattern occurred in a large hematocyst and in necrotic tissue, without sufficient blood supply. It is suggested that the contrast-enhancement pattern of hemangiosarcoma may be dependent on the formation of blood clots in the tumor.

Undifferentiated sarcoma displayed a heterogeneous normal enhancement pattern. Additionally, high enhanced volumetric ratios reflect nodular hyperplasia and malignant splenic stromal neoplasia. A similar undifferentiated sarcoma has been described as a fibrohistiocytic nodule [15]. The survival time in these cases is dependent on the proportion of nodular hyperplasia to fibrohistiocytic cells. In this study, nodular hyperplasia displayed more intense enhancement, and undifferentiated sarcoma not containing nodular hyperplasia showed lower enhanced volumetric ratios. Therefore, it is suggested that the region of contrast enhancement of fibrohistiocytic nodules contains nodular hyperplasia, and that enhanced volumetric ratios can be used as a prognostic indicator in cases with fibrohistiocytic nodules. Undifferentiated sarcoma and hematoma showed a heterogeneous normal enhancement pattern. The enhanced volumetric ratios of hematoma tended to be greater than that of undifferentiated sarcoma. However, fibrohistiocytic nodules could not be differentiated from hematoma by the use of triple-phase helical CT alone, because both masses showed high values of the enhanced volumetric ratios, representing nodular hyperplasia.

In another contrast-enhanced CT (scanned 1 min after injection) study in dogs, it was reported that nodular hyperplasia displayed the highest enhancement, followed by hematoma, with malignant splenic masses displaying the least enhancement [2]. ROIs were drawn to encompass the entire mass on the image in the previous study. In this study, there was a trend towards homogeneous iso-enhancement of nodular hyperplasia, higher enhanced volumetric ratios in hematoma, and lower enhanced volumetric ratios in malignant splenic masses (hemangiosarcoma and undifferentiated sarcoma). As hemangiosarcoma with a good arterial supply displayed higher intensities than the other splenic masses, we suggest that evaluation of enhancement based on Hounsfield units alone has a risk of erroneous differential diagnoses of splenic masses in dogs. In hemangiosarcoma, the contrast-enhancement pattern showed remarkable enhancement, with the peak contrast value in the arterial phase, decreasing thereafter to normal enhancement in the delayed phase. Thus, it is important that triple-phase helical CT is performed.

In triple-phase helical CT of the liver, scanning during the arterial and portal venous phases is important because of the dual blood supply (artery and portal vein) to the liver $[8,11]$. In contrast, the spleen receives its entire blood supply from the splenic artery, and drains into the portal vein. Therefore, we considered the portal venous phase to be a blood drainage phase. In our study, body weight and injection speed were found to have no significant correlations with any of the splenic parenchyma and mass contrast values.

MDCT makes it possible to scan a greater number of thinner slices more quickly than the conventional single-slice CT. The thinner slice makes it possible to reconstruct more exact 3D images. The enhanced volumetric ratio was calculated using the 3D reconstructed images, which showed detailed morphological features of the canine intra-abdominal structures. In addition, the 3D reconstruction imaging technique could identify large masses involving the vessels and the organs. It is therefore suggested that triple-phase helical CT provides significant information for the differential diagnosis of splenic masses, as well as for surgical planning.

In conclusion, our study demonstrated that triple-phase helical CT has the potential for differentiating between canine nodular hyperplasia, hematoma, hemangiosarcoma, and undifferentiated sarcoma. However, splenic masses in our study did not include diffuse masses (such as lymphoma, and mast cell tumor), and other solid tumors (such as stromal tumor, myelolipoma, and metastatic tumor), because there were too few of those splenic masses for statistical analysis. Further large-scale studies are warranted to explore the clinical applications of triple-phase helical CT in dogs with splenic masses.

\section{REFERENCES}

1. Akai, H., Kiryu, S., Matsuda, I., Satou, J., Takao, H., Tajima, T., Watanabe, Y., Imamura, H., Kokudo, N., Akahane, M. and Ohtomo, K. 2011. Detection of hepatocellular carcinoma by Gd-EOB-DTPA-enhanced liver MRI: comparison with triple phase 64 detector row helical CT. Eur. J. Radiol. 80: 310-315. [Medline] [CrossRef]

2. Fife, W. D., Samii, V. F., Drost, W. T., Mattoon, J. S. and Hoshaw-Woodard, S. 2004. Comparison between malignant and nonmalignant splenic masses in dogs using contrast-enhanced computed tomography. Vet. Radiol. Ultrasound 45: 289-297. [Medline] [CrossRef]

3. Hanson, J. A. and Penninck, D. G. 1994. Ultrasonographic evaluation of a traumatic splenic hematoma and literature review. Vet. Radiol. Ultrasound 35: 463-468. [CrossRef]

4. Ivancić, M., Long, F. and Seiler, G. S. 2009. Contrast harmonic ultrasonography of splenic masses and associated liver nodules in dogs. J. Am. Vet. Med. Assoc. 234: 88-94. [Medline] [CrossRef]

5. Jeon, T. Y., Kim, S. H., Lim, H. K. and Lee, W. J. 2010. Assessment of triple-phase CT findings for the differentiation of fat-deficient hepatic angiomyolipoma from hepatocellular carcinoma in non-cirrhotic liver. Eur. J. Radiol. 73: 601-606. [Medline] [CrossRef]

6. Kutara, K., Konno, T., Kondo, H., Aoki, K., Yamazoe, H. and Matsunaga, S. 2017. Imaging diagnosis -ectopic spleen mimicking hepatic tumor with intra-abdominal metastases investigated via triple-phase helical computed tomography in a dog. Vet. Radiol. Ultrasound 58: E26-E30. [Medline] [CrossRef] 
7. Kutara, K., Asano, K., Kitagawa, M., Ito, D., Teshima, K., Nakayama, T., Watari, T. and Tanaka, S. 2011. Evaluation of protocol for abdominal contrast computed tomography in small-breed dogs. J Jpn Vet Med Assoc 64: 385-389 (in Japanese). [CrossRef]

8. Kutara, K., Seki, M., Ishikawa, C., Sakai, M., Kagawa, Y., Iida, G., Ishigaki, K., Teshima, K., Edamura, K., Nakayama, T. and Asano, K. 2014. Triple-phase helical computed tomography in dogs with hepatic masses. Vet. Radiol. Ultrasound 55: 7-15. [Medline] [CrossRef]

9. Linder, K. E. 2017. Tumors of spleen. pp. 307-336. In: Tumors in Domestic Animals. 5th ed. (Meuten, D. J. ed.), Wiley Blackwell, Iowa.

10. Moss, A. A., Korobkin, M., Price, D. and Brito, A. C. 1979. Computed tomography of splenic subcapsular hematomas: an experimental study in dogs. Invest. Radiol. 14: 60-64. [Medline] [CrossRef]

11. Scialpi, M., Volterrani, L., Mazzei, M. A., Cappabianca, S., Barberini, F., Piscioli, I., Brunese, L. and Lupattelli, L. 2009. Small $(<$ or $=2 \mathrm{~cm})$ atypical hepatic haemangiomas in the non-cirrhotic patient: pattern-based classification scheme for enhancement at triple-phase helical CT. Radiol. Med. (Torino) 114: 935-947. [Medline] [CrossRef]

12. Soyer, P., Poccard, M., Boudiaf, M., Abitbol, M., Hamzi, L., Panis, Y., Valleur, P. and Rymer, R. 2004. Detection of hypovascular hepatic metastases at triple-phase helical CT: sensitivity of phases and comparison with surgical and histopathologic findings. Radiology 231: 413-420. [Medline] [CrossRef]

13. Spangler, W. L. and Culbertson, M. R. 1992. Prevalence, type, and importance of splenic diseases in dogs: 1,480 cases (1985-1989). J. Am. Vet. Med. Assoc. 200: 829-834. [Medline]

14. Spangler, W. L. and Kass, P. H. 1997. Pathologic factors affecting postsplenectomy survival in dogs. J. Vet. Intern. Med. 11: 166-171. [Medline] [CrossRef]

15. Spangler, W. L. and Kass, P. H. 1998. Pathologic and prognostic characteristics of splenomegaly in dogs due to fibrohistiocytic nodules: 98 cases. Vet. Pathol. 35: 488-498. [Medline] [CrossRef]

16. Taniura, T., Marukawa, K., Yamada, K., Hikasa, Y. and Ito, K. 2009. Differential diagnosis of hepatic tumor-like lesions in dog by using dynamic CT scanning. Hiroshima J. Med. Sci. 58: 17-24. [Medline]

17. Tidwell, A. S. 2007. Principles of computed tomography and magnetic resonance imaging. pp. 50-77. In: Textbook of Veterinary Diagnostic Radiology. 6th ed. (Thrall, D. E. ed.), Saunders Elsevier, St. Louis.

18. Wrigley, R. H., Konde, L. J., Park, R. D. and Lebel, J. L. 1989. Clinical features and diagnosis of splenic hematomas in dogs: 10 cases (1980-1987). J. Am. Vet. Med. Assoc. 25: 371-375.

19. Wrigley, R. H., Park, R. D., Konde, L. J. and Lebel, J. L. 1988. Ultrasonographic features of splenic hemangiosarcoma in dogs: 18 cases (19801986). J. Am. Vet. Med. Assoc. 192: 1113-1117. [Medline] 